\title{
Lattices of Theories in Languages without Equality
}

\author{
J. B. Nation
}

\begin{abstract}
If $\mathbf{S}$ is a semilattice with operators, then there is an implicational theory $\mathcal{Q}$ such that the congruence lattice $\operatorname{Con}(\mathbf{S})$ is isomorphic to the lattice of all implicational theories containing $\mathcal{Q}$.
\end{abstract}

The author and Kira Adaricheva have shown that lattices of quasi-equational theories are isomorphic to congruence lattices of semilattices with operators. That is, given a quasi-equational theory $\mathcal{Q}$, there is a semilattice with operators $\mathbf{S}$ such that the lattice $\operatorname{QuTh}(\mathcal{Q})$ of quasi-equational theories containing $\mathcal{Q}$ is isomorphic to $\operatorname{Con}(\mathbf{S})$. There is a partial converse: if the semilattice has a largest element 1 , and under strong restrictions on the monoid of operators, then $\operatorname{Con}(\mathbf{S},+, 0, \mathcal{F})$ can be represented as a lattice of quasi-equational theories. Any formulation of a converse will necessarily involve some restrictions, as there are semilattices with operators whose congruence lattice cannot be represented as a lattice of quasi-equational theories. In particular, one must deal with the element corresponding to the relative variety $x \approx y$, which has no apparent analogue in congruence lattices of semilattices with operators.

In this note, it is shown that if $\mathbf{S}$ is a semilattice with operators, then $\operatorname{Con}(\mathbf{S},+$, $0, \mathcal{F})$ is isomorphic to a lattice of implicational theories in a language that may not contain equality. The proof is a modification of the previous argument, Adaricheva and Nation [1], but not an entirely straightforward one. En route, we also investigate atomic theories, the analogue of equational theories for a language without equality.

For classical logic without equality, see Church [4] or Monk [12]. More recent work includes Blok and Pigozzi [2], Christie et al. [3], Czelakowski [5], and Elgueta [6]. The standard reference for quasi-varieties is Viktor Gorbunov's book [7].

The rules for deduction in implicational theories are given explicitly in Section 3. Our main result, Corollary 8 , of course depends on these. It does not depend on the model theory used to interpret how it applies to structures, and indeed, there are options in this regard. So there are two versions of this paper. The longer one

Received April 2, 2010; accepted June 3, 2012

2010 Mathematics Subject Classification: Primary 08C15; Secondary 08B15, 03G10

Keywords: quasi-variety, quasi-equational theory, congruence lattice, semilattice

(C) 2013 by University of Notre Dame 10.1215/00294527-1960470 
includes a suitably weak model theory to interpret the results, while the shorter one proceeds more directly to the main theorem. This is the short version; both are available on the author's website: www.math.hawaii.edu/ jb.

\section{Atomic Theories}

1.1 Language Let us work in a language $\mathscr{L}$ that has a set of variables $X$, constants, function symbols, relation symbols, and punctuation, but no primitive equality relation. Constants are regarded as nullary functions, but assume that $\mathscr{L}$ has no nullary relations.

1.2 Structure An $\mathscr{L}$-structure is $\mathbf{A}=\left\langle A, \mathscr{F}^{\mathbf{A}}, \mathcal{R}^{\mathbf{A}}\right\rangle$ with the following interpretation. The carrier set $A$ is nonempty. For each $k$-ary function symbol $f$, there is a function $f^{\mathbf{A}}: A^{k} \rightarrow A$. For each $k$-ary relation symbol $R$, there is a relation $R^{\mathbf{A}} \subseteq A^{k}$. Relations of $\mathbf{A}$ are allowed to be empty. A much more general interpretation is used in the long version, but this traditional view suffices for our purposes.

1.3 Congruence A congruence $\theta$ on $\mathbf{A}$ is a set of relations $R^{\theta}$ such that $R^{\theta} \supseteq R^{\mathbf{A}}$ for each relation symbol $R$. Since there is no special relation $\approx$ satisfying particular properties, that's it. Reflexivity, transitivity, and compatibility are all implications, so structures with equality properly belong in Section 3. (However, the congruence lattices of semilattices with operators in the main results are congruences in the traditional sense.)

The structure $\left\langle\mathbf{A}, \mathcal{F}^{\mathbf{A}}, \mathcal{R}^{\theta}\right\rangle$ is denoted $\mathbf{A} / \theta$.

1.4 Atomic theories As usual, form the absolutely free structure $\mathbf{F}=\mathbf{F}_{\mathscr{L}}(X)$. No relations hold on $\mathbf{F}$, but we can form $\mathcal{R}(\mathbf{F})$, the set of all potential relation instances on $\mathbf{F}$. The elements of $\mathbf{F}$ are called terms, and members of $\mathcal{R}(\mathbf{F})$ are atomic formulas.

Note that $\mathbf{F}$ is an algebra in the usual sense, and any map $\sigma: X \rightarrow \mathbf{F}$ can be extended to a homomorphism in the usual way. We refer to these endomorphisms as substitutions and use $\operatorname{Sbn}(\mathbf{F})$ to denote the monoid of all substitutions.

A subset $\Sigma \subseteq \mathcal{R}(\mathbf{F})$ is an atomic theory if whenever $R(\mathbf{t}) \in \Sigma$ and $\sigma \in \operatorname{Sbn}(\mathbf{F})$, then $R(\sigma \mathbf{t}) \in \Sigma$. That is, atomic theories are just sets of relations on $\mathbf{F}$ that are closed under substitution.

By general principles, the lattice of all atomic theories of $\mathscr{L}$ forms an algebraic lattice $\operatorname{ATh}(\mathscr{L})$.

\section{Lattices of Atomic Theories}

2.1 Fully invariant congruences and lattices of atomic theories A fully invariant congruence is a set of relations closed under substitution endomorphisms. These again form an algebraic lattice Ficon $\mathbf{F}$.

The collection of all atomic theories extending a given theory $\Sigma$ is also an algebraic lattice, denoted by $\operatorname{ATh}(\Sigma)$. Without a primitive equality, the only means of deduction for atomic formulas is substitution. Evidently, the following holds.

Theorem 1 For an atomic theory $\Sigma$, the lattice $\operatorname{ATh}(\Sigma)$ is isomorphic to Ficon $\mathbf{F}_{\Sigma}(X)$ with $X$ countably infinite.

The structure of the lattices $\operatorname{At}(\Sigma)$ is the topic of the fourth part of this series (see Holmes, Kitsuwa, Nation, and Tamagawa [8]). In particular, these lattices are completely distributive and coatomic. 


\section{Implicational Theories}

Formally, an implication is an ordered pair $\langle F, Q\rangle$ with $F$ a finite set of atomic formulas and $Q$ an atomic formula. Thus each $P \in F$ and $Q$ are of the form $A(\mathbf{t})$ with $A$ a relational symbol and $\mathbf{t} \in \mathbf{F}^{n}$. To reflect the intended interpretation, we write an implication $\langle F, Q\rangle$ with $F=\left\{P_{0}, \ldots, P_{m}\right\}$ as either $F \Longrightarrow Q$ or $\& P_{i} \Longrightarrow Q$. The antecedent is allowed to be empty: $\varnothing \Longrightarrow P$ is equivalent to $P$. The formal definition insures that the conjunction in the antecedent is idempotent, commutative, and associative.

A collection $T$ of implications is an implicational theory if

(i) $F \Longrightarrow P$ is in $T$ whenever $P \in F$;

(ii) when $F \Longrightarrow Q$ is in $T$ and $F \subseteq G$, then $G \Longrightarrow Q$ is in $T$;

(iii) whenever $F \Longrightarrow Q$ is in $T$ for all $Q \in G$, and $G \Longrightarrow R$ is in $T$, then $F \Longrightarrow R$ is in $T$;

(iv) $T$ is closed under substitutions: if $\Phi \in T$ and $\sigma: X \rightarrow \mathbf{F}$, then $\sigma \Phi \in T$.

Note that condition (iii), transitivity, implies modus ponens:

(v) if $P_{i} \in T$ for all $i$ and $\& P_{i} \Longrightarrow Q$ is in $T$, then $Q \in T$.

The free $T$-structure on $X$ is $\mathbf{F}_{\mathscr{L}}(X)$ with the purely atomic relations of $T$, denoted $\mathbf{F}_{T}(X)$. Thus $A(\mathbf{t})$ holds in $\mathbf{F}_{T}(X)$ if and only if $A(\mathbf{t})$ is in $T$.

\section{Relative Congruences}

Let $T$ be an implicational theory. A congruence $\theta$ on $\mathbf{A}$ is a $T$-congruence if $\mathbf{A} / \theta \in \operatorname{Mod} T$, that is, $\mathbf{A}$ with the relations $\theta$ satisfies all the formulas of $T$. So a congruence $\theta$, regarded as a set of relations, is a $T$-congruence if, whenever $\& P_{i} \Longrightarrow Q$ is in $T$ and $P_{i}(\alpha \mathbf{x}) \in \theta$ for some substitution $\alpha: X \rightarrow \mathbf{A}$ and all $i$, then $Q(\alpha \mathbf{x}) \in \theta$. Again, the set of all $T$-congruences on $\mathbf{A}$ forms an algebraic lattice $\operatorname{Con}_{T}(\mathbf{A})$, as the closure operator $\operatorname{con}_{T}$ is finitary in nature.

For any set $M$ of atomic formulas, let $\operatorname{con}_{T}(M)$ denote the $T$-congruence generated by $M$, that is, the smallest $T$-congruence containing $M$. Thus $\operatorname{con}_{T}(M)$ contains $M$ and all its $T$-consequences.

Consider the substitution endomorphisms of the free algebra $\mathbf{F}_{T}(X)$, that is, the homomorphisms $\varepsilon$ generated by maps $\varepsilon_{0}: X \rightarrow \mathbf{F}$. These maps form a monoid, denoted $\operatorname{Sbn}(\mathbf{F})$. (Since the relational part of an endomorphism is not determined by the substitution for the variables, $\mathbf{F}$ may have other endomorphisms.)

The substitution endomorphisms of $\mathbf{F}$ act naturally on the compact congruences of $\operatorname{Con}_{T}(\mathbf{F})$. For $\varepsilon \in \operatorname{Sbn} \mathbf{F}$, define

$$
\begin{aligned}
\widehat{\varepsilon}\left(\operatorname{con}_{T}(R(\mathbf{s}))\right) & =\operatorname{con}_{T}(R(\varepsilon \mathbf{s})), \\
\widehat{\varepsilon}\left(\bigvee_{j} \varphi_{j}\right) & =\bigvee_{j} \widehat{\varepsilon} \varphi_{j} .
\end{aligned}
$$

Lemma 2 below checks the crucial technical detail that $\widehat{\varepsilon}$ is well defined, and hence join preserving, because $\psi \leq \bigvee_{j} \varphi_{j}$ implies $\widehat{\varepsilon} \psi \leq \bigvee_{j} \widehat{\varepsilon} \varphi_{j}$ for principal congruences $\psi$ and $\varphi_{j}$ in $\operatorname{Con}_{T}(\mathbf{F})$. Also note that $\widehat{\varepsilon}$ is zero preserving: the least $T$ congruence $\Delta_{T}$ of $\mathbf{F}$ contains exactly those relations $A(\mathbf{t})$ such that $A(\mathbf{t})$ is in $T$, and $\widehat{\varepsilon}\left(\Delta_{T}\right)=\Delta_{T}$ because $T$ is closed under substitution. Let $\widehat{\mathcal{E}}=\{\widehat{\varepsilon}: \varepsilon \in \operatorname{Sbn} \mathbf{F}\}$.

The next lemma reflects the interpretation that $\operatorname{con}_{T}(M)$ consists of $M$ and all its $T$-consequences. 
Lemma 2 If $T$ is an implicational theory, then $\operatorname{con}_{T}(Q) \leq \bigvee_{i} \operatorname{con}_{T}\left(P_{i}\right)$ holds in $\operatorname{Con}_{T}(\mathbf{F})$ if and only if $\&_{i} P_{i} \Longrightarrow Q$ is in $T$.

\section{Lattices of Implicational Theories}

Form the lattice $\operatorname{ITh}(T)$ of all implicational theories extending $T$, an algebraic lattice.

Theorem 3 For an implicational theory $T$,

$$
\operatorname{ITh}(T) \cong \operatorname{Con} \mathbf{S},
$$

where $\mathbf{S}=\langle\mathbf{U}, \vee, 0, \widehat{\mathcal{E}}\rangle$ with $\mathbf{U}$ the semilattice of $T$-congruences that are compact in $\operatorname{Con}_{T}(\mathbf{F}), \mathcal{E}=\operatorname{Sbn}(\mathbf{F})$, and $\mathbf{F}=\mathbf{F}_{T}(X)$ with $|X|=\aleph_{0}$.

At one point, we use a technical variant, with the same proof.

Theorem 4 Let $T$ be an implicational theory, and let $n \geq 1$ be an integer. The lattice of all implicational theories that

(1) contain $T$, and

(2) are determined relative to $T$ by implications in at most $n$ variables

is isomorphic to Con $\mathbf{S}_{n}$, where $\mathbf{S}_{n}=\langle\mathbf{U}, \vee, 0, \widehat{\mathcal{E}}\rangle$ with $\mathbf{U}$ the semilattice of $T$ congruences that are compact in $\operatorname{Con}_{T}(\mathbf{F}), \mathcal{E}=\operatorname{Sbn}(\mathbf{F})$, and $\mathbf{F}=\mathbf{F}_{T}(n)$.

For the proof of this theorem, and for its application, it is natural to use two structures closely related to the congruence lattice instead (see [1]). For an algebra $\mathbf{A}$ with a join semilattice reduct, let Don $\mathbf{A}$ be the lattice of all reflexive, transitive, compatible relations $R$ such that $\geq \subseteq R$; that is, $x \geq y$ implies $x R y$. Let Eon $\mathbf{A}$ be the lattice of all reflexive, transitive, compatible relations $R$ such that

(1) $R \subseteq \leq$; that is, $x R y$ implies $x \leq y$, and

(2) if $x \leq y \leq z$ and $x R z$, then $x R y$.

Lemma 5 If $\mathbf{A}=\langle A, \vee, 0, \mathcal{F}\rangle$ is a semilattice with operators, then Con $\mathbf{A} \cong$ Don $\mathbf{A} \cong$ Eon $\mathbf{A}$.

The proof of the lemma is fairly straightforward and can be found in [1, Part I].

Proof Define the map $\kappa: \operatorname{ITh}(T) \rightarrow \operatorname{Don} \mathbf{S}$ by $(\theta, \psi) \in \kappa(K)$ if and only if there are $P_{0}, \ldots, P_{m}, Q_{0}, \ldots, Q_{n}$ such that

- for each $j$, the implication $\& P_{i} \Longrightarrow Q_{j}$ is in $K$,

- $\theta=\bigvee_{i} \operatorname{con}_{T}\left(P_{i}\right)$ in $\operatorname{Con}_{T}(\mathbf{F})$, and

- $\psi=\bigvee_{j} \operatorname{con}_{T}\left(Q_{j}\right)$ in $\operatorname{Con}_{T}(\mathbf{F})$.

In the other direction, define $\tau: \operatorname{Don} \mathbf{S} \rightarrow \operatorname{ITh}(T)$ such that $\& P_{i} \Longrightarrow Q$ is in $\tau(R)$ if and only if $\left(\bigvee \operatorname{con}_{T}\left(P_{i}\right), \operatorname{con}_{T}(Q)\right)$ is in $R$.

The proof of the theorem is mostly routine checking, modulo Lemma 2.

First, we check that $\kappa(K) \in$ Don $\mathbf{S}$. Reflexivity follows from property (i) of $K$.

The transitivity of $\kappa(K)$ requires some care. Suppose $\theta \kappa(K) \psi \kappa(K) \varphi$, where

$$
\begin{aligned}
\theta & =\bigvee_{i} \operatorname{con}_{T}\left(P_{i}\right), \\
\psi & =\bigvee_{j} \operatorname{con}_{T}\left(Q_{j}\right)=\bigvee_{k} \operatorname{con}_{T}\left(R_{k}\right), \\
\varphi & =\bigvee_{\ell} \operatorname{con}_{T}\left(S_{\ell}\right),
\end{aligned}
$$


with the corresponding implications being in $K$. Now $\& Q_{j} \Longrightarrow R_{k}$ is in $T$ for all $k$ by Lemma 2 , and $T \subseteq K$. Thus $\& P_{i} \Longrightarrow R_{k}$ is in $K$ for all $k$ by (iii). Apply (iii) once more to obtain $\& P_{i} \Longrightarrow S_{\ell}$ in $K$ for all $\ell$, whence $\theta \kappa(K) \varphi$.

The compatibility of $\kappa(K)$ with join, $\theta \kappa(K) \psi$ implies $\theta \vee \varphi \kappa(K) \psi \vee \varphi$, follows from conditions (i) and (ii). Compatibility with substitutions is condition (iv).

That $\theta \geq \psi$ implies $\theta \kappa(K) \psi$ follows from Lemma 2 and $T \subseteq K$.

We conclude that $\kappa(K) \in$ Don $\mathbf{S}$. It is also clear that $\kappa$ is order preserving.

Next, given $R \in$ Don $\mathbf{S}$, check that $\tau(R)$ is an implicational theory. Properties (i) and (ii) follow from $\geq \subseteq R$ and the transitivity of $R$. For property (iii), note that if $\left(\bigvee_{i} \operatorname{con}_{T}\left(P_{i}\right), \operatorname{con}_{T}\left(Q_{j}\right)\right) \in R$ for all $j$, then $\left(\bigvee_{i} \operatorname{con}_{T}\left(P_{i}\right), \bigvee_{j} \operatorname{con}_{T}\left(Q_{j}\right)\right) \in R$ since $R$ is compatible with respect to joins. If in addition $\left(\bigvee_{j} \operatorname{con}_{T}\left(Q_{j}\right)\right.$, $\left.\operatorname{con}_{T}(S)\right) \in R$, then $\left(\bigvee_{i} \operatorname{con}_{T}\left(P_{i}\right), \operatorname{con}_{T}(S)\right) \in R$ by the transitivity of $R$. Finally, closure under substitution, (iv), is immediate from the definition of $\widehat{\varepsilon}$.

Moreover, $\tau(R) \supseteq T$ by Lemma 2, and $\tau$ is order preserving.

Finally, using Lemma 2 again, we note that $\kappa \tau(R)=R$ and $\tau \kappa(K)=K$ for all appropriate $R$ and $K$.

Recall that there is a natural equa-interior operator on lattices of quasi-equational theories. Given a quasi-equational theory $\mathcal{Q}$ and a theory $\mathcal{T}$ in $\operatorname{QuTh}(\mathcal{Q})$, define $\eta(\mathcal{T})$ to be the implicational theory generated by $\mathcal{Q}$ and all the equations valid in $\mathcal{T}$. This interior operator has the following properties (see [7]):

(I1) $\eta(x) \leq x$.

(I2) $x \geq y$ implies $\eta(x) \geq \eta(y)$.

(I3) $\eta^{2}(x)=\eta(x)$.

(I4) $\eta(1)=1$.

(I5) $\eta(x)=u$ for all $x \in X$ implies $\eta(\bigvee X)=u$.

(I6) $\eta(x) \vee(y \wedge z)=(\eta(x) \vee y) \wedge(\eta(x) \vee z)$.

(I7) The image $\eta(\mathbf{L})$ is the complete join subsemilattice of $\mathbf{L}$ generated by $\eta(\mathbf{L}) \cap \mathbf{L}_{c}$.

(I8) There is a compact element $w \in \mathbf{L}$ such that $\eta(w)=w$ and the interval $[w, 1]$ is isomorphic to the congruence lattice of a semilattice. Thus the interval $[w, 1]$ is coatomistic.

In view of (I5), let $\tau(x)=\bigvee\{z: \eta(z)=\eta(x)\}$. A ninth property was added in [1].

(I9) For any index set $I$, if $\eta(x) \leq c$ and $\bigwedge \tau\left(z_{i}\right) \leq \tau(c)$, then $\eta(\eta(x) \vee$ $\left.\bigwedge_{i \in I} \tau\left(x \wedge z_{i}\right)\right) \leq c$.

There is also a natural interior operator defined on the congruence lattice of any semilattice with operators, where $\eta(\theta)$ is the congruence generated by the 0 -class of $\theta$. This operator satisfies properties (I1)-(I7) and (I9). However, it need not satisfy (I8), which for lattices of quasi-equational theories refers to the relative variety determined by $x \approx y$.

These ideas fit into our current setting thusly. Let $\operatorname{ATh}^{*}(T)$ denote the lattice of implicational theories generated by $T$ and a set of purely atomic formulas. Note that $\operatorname{ATh}^{*}(T)$ is a complete join subsemilattice of $\operatorname{ITh}(T)$.

In the representation of Theorem 3, relatively atomic theories of $T$ correspond to congruences $\eta(I)$ with $I$ an $\widehat{\mathscr{E}}$-closed ideal of $\mathbf{S}$. Thus $\operatorname{ATH}^{*}(T)$ is isomorphic to the lattice $\eta(\operatorname{Con} \mathbf{S})$ for the natural interior operator, which in turn is isomorphic to the lattice of $\widehat{\mathcal{E}}$-closed ideals of $\mathbf{S}$. In particular, $\operatorname{ITh}(T)$ has a natural interior operator 
satisfying properties (I1)-(I7) and (I9), and all the consequences of that apply (see [1]).

Under the circumstances, the special role of property (I8) for implicational theories in languages with equality invites further analysis.

\section{Restoring Equality}

At this point, we pause to note that $T$ could contain implications saying that a binary relation $\approx$ is an equivalence relation and, moreover, a congruence in the usual sense. That is, $T$ could contain the laws

(1) $x \approx x$,

(2) $x \approx y \Longrightarrow y \approx x$,

(3) $x \approx y \& y \approx z \Longrightarrow x \approx z$,

(4) $x \approx y \Longrightarrow f(x, \mathbf{z}) \approx f(y, \mathbf{z})$ for all functions $f$,

(5) $x \approx y \Longrightarrow(R(x, \mathbf{z}) \Longleftrightarrow R(y, \mathbf{z}))$ for all predicates $R$.

This relation can then be regarded as equality. In this case, $T$-congruences correspond to regular congruences, $\operatorname{EqTh}(T) \cong \operatorname{ATh}^{*}(T)$ and $\operatorname{QuTh}(T) \cong \operatorname{ITh}(T)$.

Bjørn Kjos-Hanssen points out that while there may be no such relation, there is at most one, in view of (5).

\section{Representation}

Now we provide a converse to Theorem 3 .

Theorem $6 \quad$ Let $\mathscr{B}$ be an implicational theory in a language $\mathscr{L}$ with the following restrictions and laws.

(1) $\mathscr{L}$ has only unary predicate symbols.

(2) $\mathscr{L}$ has only unary function symbols.

(3) L has one constant symbol e.

(4) $B$ contains the laws $P(f(e))$ for every predicate $P$ and every formal composition $f$ of functions of $\mathscr{L}$.

Then every implication holding in a theory extending the theory of $\mathscr{B}$ is equivalent (modulo the laws of $\mathscr{B}$ ) to a set of implications in only one variable. Hence the lattice of theories of $\mathcal{B}$ is isomorphic to $\operatorname{Con}(\mathbf{S})$, where $\mathbf{S}=\langle\mathbf{T}, \vee, 0, \widehat{\mathcal{E}}\rangle$ with $\mathbf{T}$ the semilattice of compact congruences of $\operatorname{Con}_{\mathcal{B}}(\mathbf{F}), \mathcal{E}=\operatorname{Sbn}(\mathbf{F})$, and $\mathbf{F}=\mathbf{F}_{\mathcal{B}}(1)$.

Proof The atomic formulas of $\mathscr{L}$ are of the form $A(h(u))$, where $A$ is a predicate, $h$ is a formal composition of functions, possibly empty, and $u$ is a variable or $e$. In an implication $\& P_{i} \Longrightarrow Q$, the conclusion involves at most one variable. A law that is equivalent, modulo the laws of $\mathscr{B}$, is obtained by replacing every other variable occurring in the antecedent by $e$.

Theorem $7 \quad$ Let $\mathbf{S}$ be a join semilattice with 0 , and let $\mathbf{M}$ be a monoid of operators acting on $\mathbf{S}$. Then there is an implicational theory $C_{\text {such }}$ that $\operatorname{Con}(\mathbf{S},+, 0, \mathbf{M})$ is isomorphic to $\operatorname{Con}(\mathbf{T}, \vee, 0, \widehat{\mathcal{E}})$ with $\mathbf{T}$ the semilattice of compact congruences of Cone $(\mathbf{F}), \mathcal{E}=\operatorname{Sbn}(\mathbf{F})$, and $\mathbf{F}=\mathbf{F e}_{e}(1)$.

Proof Our language will include unary predicates $A$ for each nonzero element $a$ of $\mathbf{S}$, unary operations $f$ for each $f \in \mathbf{M}$, and a constant $e$.

Again, $\mathscr{L}$-terms are of the form $A(h(u))$, where $A$ is a predicate, $h$ is a formal composition of functions, and $u$ is a variable or $e$. Denote the single variable by $x$. 
The construction begins by assigning a set of predicates to each nonzero element of $\mathbf{S}$. For each $a \in \mathbf{S}$ and formal composition $h=f_{1} \cdots f_{k}$, assign the predicate $A(h(x))$ to $h^{\star}(a)$, where $h^{\star}$ denotes $h$ evaluated in $\mathbf{M}^{\text {opp }}$, that is, $h^{\star}=f_{k} \cdots f_{1}$. In this way each element of $\mathbf{S}$ may be assigned multiple predicates, but they will all be of the form $B(g(x))$ for different predicates $B$ and sequences $g$. For $s \in \mathbf{S}$, let $\mathcal{P}(s)$ denote the set of predicates assigned to $s$. Thus $\mathcal{P}(s)=\left\{A(h(x)): h^{\star}(a)=s\right\}$.

Define $\mathcal{C}$ to be the quasi-variety determined by these laws.

(1) $P(f(e))$ for every predicate $P$ and every formal composition $f$ of functions of $\mathscr{L}$.

(2) $A(i(x)) \Longleftrightarrow A(x)$ for every $A$, where $i$ is the identity element of $\mathbf{M}$.

(3) $A(h(x)) \Longleftrightarrow A\left(h^{\star}(x)\right)$ for every formal composition.

(4) $\beta \Longrightarrow \alpha$ whenever $a \leq b, \alpha \in \mathcal{P}(a), \beta \in \mathcal{P}(b)$.

(5) $\& \beta_{j} \Longrightarrow \alpha$ whenever $a \leq \sum b_{j}, \alpha \in \mathcal{P}(a), \beta_{j} \in \mathcal{P}\left(b_{j}\right)$ for each $j$.

The laws (1) ensure that Theorem 6 applies, so that we may work with $\mathbf{F}_{\mathcal{C}}(1)$. Note that the laws (4) are redundant as a special case of (5).

The universe of $\mathbf{F}=\mathbf{F}_{e}(1)$ is all terms $h(u)$ with $h$ a sequence of operations, and $u$ either $x$ or $e$. The operations correspond to elements of $\mathbf{M}$, and there is a unary predicate for each nonzero element of $\mathbf{S}$. Note that $A(t)$ holds in the free structure only for $t=e$ or $t=h(e)$. The substitution endomorphisms of $\mathbf{F}$ are determined by the image of $x$. For a term $t$, let $\varepsilon_{t}$ denote the endomorphism with $x \mapsto t$.

Since $\ell$ satisfies $\& \beta_{j} \Longrightarrow \alpha$ whenever $a \leq \sum b_{j}, \alpha \in \mathcal{P}(a)$, and each $\beta_{j} \in \mathcal{P}\left(b_{j}\right)$, we see that every $\ell$-congruence of $\mathbf{F}$ is the set of predicates assigned to some ideal of $\mathbf{S}$, along with $A(h(e))$ for every $A$ and $h$. Conversely, every ideal determines such a $\mathcal{C}$-congruence, and principal ideals determine compact congruences. In fact, the congruence corresponding to $\downarrow s$ is con $\mathcal{C}(\alpha)$ for any $\alpha \in \mathcal{P}(s)$. Thus the semilattice of compact $\ell_{\text {-congruences of }} \mathbf{F}_{\boldsymbol{C}}(1)$ is isomorphic to $\mathbf{S}$, as desired.

As a matter of notation, let $\Theta_{s}$ denote the congruence that has all the relations $\bigcup\{\mathcal{P}(t): t \leq s\}$, plus the base relations of the form $B(g(e))$ given by (1). Denote the set of base relations by $\mathbb{B}$. Thus $\Theta_{s}=\left\{A(h(x)): h^{\star}(a) \leq s\right\} \cup \mathbb{B}$.

It remains to show that the action of $\widehat{\mathscr{E}}$ on $\mathbf{T}$, the semilattice of compact $\mathcal{C}$ congruences of $\mathbf{F}_{\mathcal{C}}(1)$, mimics the action of $\mathbf{M}^{\text {opp }}$ on $\mathbf{S}$. The relevant facts are these:

- $\widehat{\varepsilon}_{h(e)}\left(\Theta_{s}\right) \subseteq \mathbb{B}$ for any $h$, whence $\mathbb{B}$ is the zero congruence, so this operator does not affect the congruences of $\mathbf{T}$.

- $\widehat{\varepsilon}_{h(x)}\left(\Theta_{s}\right)=\Theta_{h^{\star}(s)}$ for any sequence $h$ and element $s$.

- If $f$ and $g$ are sequences, then $\widehat{\varepsilon}_{f(x)}=\widehat{\varepsilon}_{g(x)}$ if and only if $\Theta_{f^{\star}(s)}=\Theta_{g^{\star}(s)}$ for all $s$, if and only if $f^{\star}=g^{\star}$.

- $\widehat{\varepsilon}_{f(x)} \widehat{\varepsilon}_{g(x)}=\widehat{\varepsilon}_{(g f)(x)}$.

The crucial calculation is the second one. Note that

$$
\begin{aligned}
\widehat{\varepsilon}_{h(x)}\left(\Theta_{s}\right) & =\operatorname{con}_{\mathcal{C}}\left\{\varepsilon_{h(x)} A(f(x)): f^{\star}(a) \leq s\right\} \cup \mathbb{B} \\
& =\operatorname{con} \mathcal{}\left\{A(f(h(x))): f^{\star}(a) \leq s\right\} \cup \mathbb{B} .
\end{aligned}
$$

Now $f^{\star}(a) \leq s$ implies $(f h)^{\star}(a)=h^{\star} f^{\star}(a) \leq h^{\star}(s)$, so $\widehat{\varepsilon}_{h(x)}\left(\Theta_{s}\right) \subseteq \Theta_{h^{\star}(s)}$. But the left-hand side includes $\varepsilon_{h(x)} S(x)=S(h(x))$, and that is a generator for $\Theta_{h^{\star}(s)}$, whence equality holds.

This completes the proof of the theorem. 
Combining these results (which now avoid problems that occurred in the presence of equality in [1, Part II]), we obtain the desired result.

Corollary $\mathbf{8} \quad$ Let $\mathbf{S}$ be a join semilattice with 0 , and let $\mathbf{M}$ be a monoid of operators acting on $\mathbf{S}$. Then there is an implicational theory $\mathcal{C}$ such that the lattice of implicational theories of $\mathcal{C}$ is isomorphic to $\operatorname{Con}(\mathbf{S},+, 0, \mathbf{M})$.

\section{Overview}

It is useful to step back and consider the situation from a distance. There are (at least) four plausible settings:

(IA) algebras, language with equality;

(IB) pure relational structures, language with equality;

(II) structures with functions and relations, language with equality;

(III) structures with functions and relations, language without equality.

Likewise, there are three types of theories:

(1) atomic theories $\operatorname{ATh}(T)$;

(2) implicational theories $\operatorname{ITh}(T)$;

(3) relative atomic theories $\mathrm{ATh}^{*}(T)$.

That makes twelve combinations, not all equally interesting.

The traditional setting for equational theories is algebras (IA). There we have the results of McKenzie [11], Newrly [13], and Nurakunov [14] leading to Lampe's zipper condition (see [9], [10]) and its generalizations. Are there any versions of this that apply in other settings?

The historic setting for quasi-equational theories is general structures (II), though pure relational structures (IB) played a role. The results of [1] are the apparent analogues here, and in this note we see how this generalizes to the setting (III). The ultimate goal is still to deal with case (II) and to discern what is special about the quasi-equational theory of algebras (IA).

Atomic theories of structures with equality (II) can be viewed as relative atomic theories of structures without equality (III). This seems an odd viewpoint, but perhaps it explains some of the complexity of lattices of equational theories.

\section{References}

[1] Adaricheva, K., and J. B. Nation, "Lattices of quasi-equational theories as congruence lattices of semilattices with operators, I," International Journal of Algebra and Computation, vol. 22, no. 7 (2012), 27 pp. DOI 10.1142/S0218196712500658. "II," International Journal of Algebra and Computation, vol. 22, no. 7 (2012), 19 pp. DOI 10.1142/S021819671250066X. 167, 170, 171, 172, 174

[2] Blok, W. J., and D. Pigozzi, "Algebraic semantics for universal Horn logic without equality," pp. 1-56 in Universal Algebra and Quasigroup Theory (Jadwisin, Poland, 1989), vol. 19 of Research and Exposition in Mathematics, Heldermann, Berlin, 1992. MR 1191226. 167

[3] Christie, A., Q. Wang, and S. L. Wismath, “The inflation class operator," Algebra Universalis, vol. 56 (2007), pp. 107-18. Zbl 1115.08002. MR 2280442. DOI 10.1007/s00012006-1988-7.

[4] Church, A., Introduction to Mathematical Logic, reprint of the 2nd (1956) edition, Princeton University Press, Princeton, 1996. MR 1435972. 167 
[5] Czelakowski, J., Protoalgebraic Logics, vol. 10 of Trends in Logic-Studia Logica Library, Kluwer, Dordrecht, 2001. Zbl 0984.03002. MR 1828895. 167

[6] Elgueta, R., "Characterizing classes defined without equality," Studia Logica, vol. 58 (1997), pp. 357-94. MR 1460253. DOI 10.1023/A:1004978316495. 167

[7] Gorbunov, V. A., Algebraic Theory of Quasivarieties, translated from the Russian, Siberian School of Algebra and Logic, Consultants Bureau, New York, 1998. MR 1654844. 167, 171

[8] Holmes, T., D. Kitsuwa, J. B. Nation, and S. Tamagawa, "Lattices of atomic theories in a language without equality," preprint, 2009.

[9] Lampe, W. A., "A property of the lattice of equational theories," Algebra Universalis, vol. 23 (1986), pp. 61-69. Zbl 0604.08003. MR 0873596. DOI 10.1007/BF01190912. 174

[10] Lampe, W. A., "Further properties of lattices of equational theories," Algebra Universalis, vol. 28 (1991), pp. 459-86. Zbl 0756.08006. MR 1128384. DOI 10.1007/BF01195857. 174

[11] McKenzie, R., "Finite forbidden lattices," pp. 176-205 in Universal Algebra and Lattice Theory (Puebla, Mexico, 1982), vol. 1004 of Lecture Notes in Mathematics, Springer, Berlin, 1983. MR 0716183. DOI 10.1007/BFb0063438. 174

[12] Monk, J. D., Mathematical Logic, vol. 37 of Graduate Texts in Mathematics, Springer, New York, 1976. Zbl 0354.02002. MR 0465767. 167

[13] Newrly, N., "Lattices of equational theories are congruence lattices of monoids with one additional unary operation," Algebra Universalis, vol. 30 (1993), pp. 217-20. Zbl 0789.08006. MR 1223629. DOI 10.1007/BF01196092. 174

[14] Nurakunov, A. M., "Equational theories as congruences of enriched monoids," $A l$ gebra Universalis, vol. 58 (2008), pp. 357-72. Zbl 1151.08003. MR 2415287. DOI 10.1007/s00012-008-2080-2. 174

\section{Acknowledgments}

This research was supported in part by U.S. Civilian Research and Development Foundation grant KYM1-2852-BI-07. The author would like to thank the University of Hawaii algebra and logic seminar for constructive comments and suggestions.

Department of Mathematics

University of Hawaii

Honolulu, Hawaii 96822

USA

jb@math.hawaii.edu 